Psicologia Escolar

e Educacional
ARTí́cULO

DOI: http://dx.doi.org/10.1590/2175-35392021220783

Localizador - e220783

\title{
HEGEMONÍA Y EMANCIPACIÓN, ELEMENTOS REPRESENTACIONALES DEL OBJETO SOCIAL COLEGIO
}

\author{
Juan Carlos Chaves-Yepes ${ }^{1} \mathbb{D}$
}

\section{RESUMEN}

La escuela, como espacio de relaciones o como establecimiento educador por la transmisión de conocimientos, mantiene una relación directa con las familias. Este estudio problematiza la relación escuela / familia en grados escolares superiores de secundaria en el contexto de la Ciudad de Pasto - Colombia. El objetivo es explorar las representaciones sociales que sobre el colegio tienen padres y madres de familia de estudiantes de secundaria. Se cuenta con información de 143 personas vinculadas a instituciones educativas públicas. Con el uso de un cuestionario de preguntas abiertas se indagó la dimensión de información y la dimensión de actitud del contenido representacional. Los resultados muestran tres categorías: desempeño de los docentes, enseñanza y desempeño de estudiantes como parte del contenido representacional, las cuales median en la imagen del colegio. Estos resultados permiten inferir que existen elementos tendientes a la conformación de representaciones sociales hegemónicas y representaciones sociales emancipadas.

Palabras clave: representación social; familia; escuela; profesores.

\section{Hegemony and emancipation, representational elements of the social object school}

\begin{abstract}
The school, as a space for relationships or as an educational establishment for the transmission of knowledge, maintains a direct relationship with families. This study problematizes the school / family relationship in upper secondary school grades in the context of the city of Pasto - Colombia. The objective is to explore the social representations that parents of high school students have about the school. It has information of 143 people linked to public educational institutions. With the use of a questionnaire of open questions, the dimension of information and the attitude dimension of the representational content were investigated. The results show three categories: teacher performance, teaching and student performance as part of the representational content, which mediate the image of the school. These results allow us to infer that there are elements tending to the conformation of hegemonic social representations and emancipated social representations.
\end{abstract}

Keywords: social representation; family; school; teachers.

\section{Hegemonia e emancipação, elementos representacionais do objeto social da escola}

\section{RESUMO}

A escola, como espaço de relações ou como estabelecimento educador pela transmissão de conhecimentos, mantém uma relação direta com as famílias. Este estudo problematiza a relação escola / família em grau escolar - ensino médio - no contexto da Cidade de Pasto - Colômbia. O objetivo é explorar as representações sociais que a escola tem pais e mães de família de estudantes de secundaria (ensino médio). Conta-se com informação de 143 pessoas vinculadas a instituições educativas públicas. Com o uso de um questionário de perguntas abertas indagou-se a dimensão de informação e a dimensão de atitude do conteúdo representacional. Os resultados mostram três categorias: desempenho dos docentes, ensino e desempenho de estudantes como parte do conteúdo representacional, as quais mediam na imagem da escola. Estes resultados permitem inferir que existem elementos tendentes à conformação de representações sociais hegemônicas e representações sociais emancipadas.

Palavras-chave: representação social; família; escola; professores.

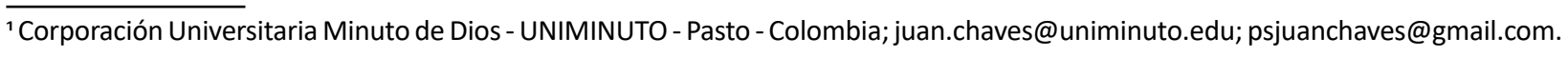




\section{INTRODUCCIÓN}

Gadotti (2003) menciona que la escuela surge desde la división social del trabajo como institución formal. La división del trabajo hizo que se piense en la especialización de las personas, por tanto, la escuela era el lugar donde unos enseñan y otros aprenden. Entre los siglos XVIII y XIX en Europa la reforma educativa implicó pasar de la educación dada dentro de los hogares a la educación dada en los centros educativos, cambio que para aquella época tuvo que ver con el pensar que la escuela era para aquellos niños cuyos padres no contaban con las capacidades intelectuales y/o económicas y de responsabilidad para educarlos (Tyrell \& Vanderstraeten, 2017). A partir de la edad moderna la escuela fue delegada como lugar del saber, como espacio de formalización e institucionalización de la enseñanza, como parte de un sistema de enseñanza formal (Silveira \& Wagner, 2009).

Si bien, la escuela mantiene una imagen de espacio primordial para la transmisión y generación de nuevos conocimientos y saberes de forma gradual, desde una visión socio histórica es entendida como un espacio diversificado de significados históricos, emocionales y sociales que permean el proceso de aprendizaje, es decir, no es solo un lugar para estudiar porque en ella se gestan dinámicas de interacción que conllevan a pensarla, más allá de un espacio físico estructural, como un espacio de encuentro, de relaciones fruto de la historia y proyectos de sus agentes (Gadotti, 2007; Araújo, Pessoa, Fonseca, Albuquerque, \& Almeida, 2016). Es el segundo ambiente mediador entre individuo y sociedad, fundamental en la socialización, el desarrollo y aprendizaje de los sujetos al considerar por un lado nuevas formas de interacción, comportamientos, y por otro la transmisión del saber culturalmente organizado, construido y sistematizado históricamente (Oliveira \& Marinho-Araújo, 2010; Pacheco \& Cia, 2015).

Si la escuela es el segundo ambiente mediador entre el individuo y sociedad, la familia es el primero, puesto que en ella desde el nacimiento se instauran en el sujeto normas, creencias, valores y roles que influyen en el desarrollo de la persona y su posterior toma de decisiones (Barboza-Palomino \& et al., 2017; Callegaro, Wanderley, \& Koller, 2015; Pacheco \& Cia, 2015). Además, la familia cobra importancia para la pedagogía por la diferenciación en el establecimiento de roles en el proceso de enseñanza / aprendizaje de los niños y niñas, e implementación de espacios alternativos de formación (Suarez \& Urrego, 2014; Tyrell \& Vanderstraeten, 2017).

Entonces se evidencia que hay un objetivo común entre familia y escuela: el bienestar de los niños, niñas, adolescentes y jóvenes, traducido en el éxito escolar y académico, lo que implica trabajar en conjunto, hacia una misma dirección (Cabello \& Giró, 2016), porque como lo plantea Gadotti (2007), la escuela "como institu- ción social depende de la sociedad y para transformarse, depende también de las relaciones que mantenga con otras escuelas, con las familias, de aprender conjuntamente con ellas, de establecer alianzas con la sociedad, con la población" (p. 10). Familia y escuela mantienen una relación que para algunos puede ser percibida como buena, cordial y fluida, mientras que para otros puede resultar problemática al no darse como se espera (Martínez, 2014).

Resultados de investigaciones muestran, por ejemplo, que una adecuada relación familia-escuela beneficia a los alumnos en aspectos como fortalecimiento de la autoestima, seguridad, confianza en sus capacidades, así mismo, favorece la iniciativa y participación en el aula destacando que una buena relación mejora el rendimiento académico de los alumnos (Castro-Zubizarreta \& Garcia-Ruiz, 2016). Y si se abordan temas específicos como el Bullying, "la implicación de los padres para la resolución de problemas puede constituirse como factor protector para los niños, disminuyendo las posibilidades de nuevas victimizaciones" (Callegaro et al., 2015, p. $45)$, es decir, tanto padres y madres y profesores como actores protagónicos del proceso formativo de los niños, pueden generar espacios en el contexto escolar y familiar para propiciar procesos de resiliencia (Cabrera, Aya, Muñoz, Guevara, \& Cano, 2016). Sin embargo, también se encuentran resultados que muestran rupturas sustanciales, identificando familias que cumplen con aspectos meramente administrativos como matricula, recepción de informes o acudir a los llamados institucionales (Julio, Mánuel, \& Navarro, 2012), y también una participación muy baja de los padres en función de sus actividades cotidianas y del trabajo (Gutiérrez \& Alonso, 2011).

Teniendo en cuenta específicamente lo que padres y madres de familia refieren respecto a la escuela, Bustamante (2010) en su estudio llevado a cabo en Córdoba capital (Argentina) menciona que para estos actores la escuela es un espacio trascendental en la vida de sus hijos, dándole valor a su estatus socializador y esperando que su función disciplinaria se geste, sin embargo algunos ven a la escuela fragmentada, lo cual opera en la valoración que tienen de ella a partir de parámetros que refuerzan la reproducción de desigualdades. Y esto se complementa con lo expuesto por Villarroel y Sánchez (2002) quienes en su estudio en el sector rural (en Valparaiso, Chile) mencionan que en los primeros años (1 a 6 grado) tanto estudiantes como padres de familia le atribuyen a la escuela una gran importancia, generando expectativas educacionales altas.

Resultados como los presentados dejan ver que estudiar la relación entre escuela y familia representa una fuente importante de información "pues permite identificar aspectos o condiciones que influencian en la comunicación, los patrones de colaboración y los conflictos entre estas dos instituciones (Pacheco \& Cia, 
2015, p. 350), y así lo reafirma De Freitas (2008) cuando menciona que "la investigación de las diferentes prácticas, estilos y comportamientos de los grupos familiares en relación al universo escolar ha permitido comprender mejor la constitución de distintas trayectorias escolares o formas de escolaridad" (p. 386).

Teniendo en cuenta lo anterior, el propósito de este estudio es aproximarse a esa relación desde un contexto particular, la ciudad de Pasto (Colombia) y particularmente con padres y madres de familia cuyos hijos e hijas se encuentran en grados superiores de escuela secundaria.

El estudio problematiza el tema desde un marco psicosocial teniendo en cuenta el concepto de Representaciones Sociales o conocimiento de sentido común, el cual se estructura desde las interacciones sociales que se producen y circulan dentro de la cotidianidad a través de una palabra, un gesto o un encuentro, resultan de observaciones y de análisis de estas observaciones, de la apropiación de nociones y lenguajes, y tienen como función permitirles a los sujetos posicionarse frente a objetos sociales definidos enmarcando sus conductas (Moscovici, 1979). Representar una cosa o un estado es reconstruirlo, retocarlo, cambiarle el texto, no solamente repetirlo o reproducirlo (Moscovici, 2000). Para Jodelet (2011) "esta forma de conocimiento tiene una raíz y un objetivo práctico: apoyándose en la experiencia de las personas, sirve de grilla de lectura de la realidad y de guía de acción en la vida práctica y cotidiana" (p.134). Dichas representaciones contienen tres dimensiones: a) información, que tiene que ver con los conocimientos que el sujeto tiene respecto al objeto, b) actitud, que tiene que ver con la posición de favorabilidad o desfavorabilidad que el sujeto tiene hacia el objeto, y c) campo de representación, que tiene que ver con la organización de los anteriores elementos estableciendo redes entre sí mismos (Jodelet, 1993; Moscovici, 1979).

El consenso que existe en los elementos de la representación dentro de un conjunto poblacional no implica uniformidad, Moscovici (1988) menciona que existen tres formas en que una representación se convierte en social teniendo en cuenta las relaciones entre los miembros de un grupo: a) las representaciones hegemónicas, compartidas por todos los miembros de un grupo estructurado, pero estas no han sido producidas por ellos, éstas son implícitas en las prácticas y se caracterizan por su uniformidad y su orden coercitivo; b) representaciones emancipadas, cuando cada subgrupo crea una versión propia y diferente de esas representaciones y las comparte con los demás; y c) las representaciones polémicas, que como producto de conflictos sociales y controversias, la sociedad en su conjunto no las comparte, y generan relaciones antagónicas entre sus miembros puesto que pretenden ser excluyentes, se consideran dentro de contextos de oposición y lucha entre grupos.

En consecuencia, para la elaboración de este texto se generó como interrogante ¿qué consensos entre elementos representacionales acerca del colegio se pueden delinear desde las expresiones de padres y madres de familia de estudiantes de los grados noveno, décimo y once de instituciones educativas del sector urbano y rural de la ciudad de Pasto? Así, se tiene como objetivo explorar los consensos entre elementos representacionales que sobre el colegio tienen padres y madres de familia de estudiantes de grados superiores de escuela secundaria, urbana y rural, en la Ciudad de Pasto.

\section{MÉTODO}

Se tomó como población a los padres y madres de familia cuyos hijos, para el momento de la recolección de datos (septiembre a noviembre 2017), se encontraban cursando grado noveno, décimo y once, pertenecientes a dos instituciones educativas públicas del sector urbano de la ciudad de Pasto, y una del sector rural, las cuales fueron seleccionadas bajo el criterio de conveniencia puesto que ya había contacto preliminar con actores claves en ellas, y se contó con las respectivas autorizaciones institucionales.

El presente artículo muestra resultados cualitativos generados a partir de una actividad de acercamiento con la población de padres y madres de familia en el marco de las actividades de escuela de familia que desarrollan las Instituciones Educativas desde su plan de trabajo, y en la cual participaron 167 personas.

Del total de participantes, para la selección de la unidad de trabajo se aplicó el siguiente criterio de selección: ser padre o madre de familia de los niños y niñas de las instituciones educativas, descartando así otro tipo de parentesco (abuelo(a), tío(a), hermano(a), otro). Como resultado se obtuvo una muestra definitiva de 143 personas. De ellos, 126 (88\%) son madres de familia y 17 (12\%) son padres de familia; el $20 \%$ de participantes están vinculados a una institución del sector rural y el $80 \%$ hacen parte de las dos instituciones del sector urbano.

En los encuentros institucionales se le dio a conocer el objetivo del proyecto de investigación en curso, y se solicitó la participación voluntaria en el mismo aclarando que los datos recolectados son anónimos guardando la confidencialidad de estos. Una vez recibido el aval verbal de los participantes se procedió a realizar la recolección de información basándose en la técnica utilizada por Diaz y Salamanca (2012) para el abordaje del contenido de las representaciones sociales abarcando las dimensiones: información y actitud. El instrumento consistió en formular preguntas específicas respecto al objeto social de estudio así: ¿qué dicen cuando hablan del colegio? ¿qué escuchan decir sobre el colegio? ¿qué es lo que les gusta y les disgusta del colegio? ¿cómo parti- 
cipan en el colegio? Estas preguntas se formularon en plenaria y los participantes consignaron sus respuestas de manera escrita, para el caso de la zona urbana en un formato de cinco casillas libres, una casilla para cada respuesta, y en el caso de la zona rural en notas adhesivas que fueron ubicando en un mural. Estas dinámicas tuvieron que ver con los diferentes contextos procurando comodidad y facilidad para los participantes.

Los datos recolectados fueron analizados bajo un proceso de codificación y categorización cualitativa y se recurrió al uso del software de análisis de datos cualitativos Atlas.ti 8. La codificación se hizo a partir de: lo dicho y lo escuchado como elementos de la categoría información, y con gusto/disgusto y participación como elementos de la categoría actitud.

\section{RESULTADOS Y DISCUSIÓN}

Los resultados obtenidos dejan ver que la imagen de colegio para padres y madres de familia se proyecta desde elementos representacionales que tienen que ver por un lado con lo dicho y escuchado acerca del colegio en la dimensión de información, y el gusto o disgusto por el colegio en la dimensión de actitud.

En ambos contextos, rural y urbano, la dimensión información se moviliza bajo las siguientes sub categorías: a) desempeño docente, con fragmentos textuales ${ }^{1}$ como: "Cuando yo hablo del colegio de mi hijo son cosas muy buenas, porque tiene muy buenos maestros" (R:6), "que aveces no les gusta por que hay profesores malgeniados" (R:20), "colegio del ijo es muy bueno, excelente, muy buenos los señores profesores" (U:22), "Los alunos dicen que a veces los profesores no les escuchan" (U:29); b) enseñanza aprendizaje, con fragmentos textuales como: "Para mi el colejio es un buen colejio porque los estudiantes an avansado mucho en el aprendisaje" (R:12), "hay estudiantes que dicen que hay profesores que en algunas materias no enseñan lo que deberian enseñar" (R:22) "cuando me refiero al colegio siempre me ha causado una buena impresión por que esta alcanzando estandares buenos en educación" (U:13), "Que es buena la enseñansa del colejio apara nuestros hijo" (U:16), "mis besino dicen que aprenden mucho sus hijos" (U:29); y c) desempeño de los estudiantes, con fragmentos textuales como: "Ay personas que piensan que el colegio es malo porque algunos estudiantes son irresponsables no aprobechan por eso es malo pero eso no es asi" (R:10), "a otras personas la eh escuchado dicen q'unos alumnos son muy problemáticos" (R:19), "Que es buen colegio, tiene buenos estudiantes" (U:30), "yo e escuchado que hay algunos estudiantes que consumen sicoactivos y algunos estudiantes son muy irrespetuosos

\footnotetext{
${ }^{1}$ La transcripción de estos fragmentos, los cuales soportan los resultados, guarda fidelidad con lo expresado por los y las participantes del estudio, desde su dialecto y formas de escritura.
}

con los docentes y las directivas" (U:39), "Es malo, no exigen, hay mucho gamín... hay mucho joven en problemas de adiccion y embarazos..." (U:40).

Los fragmentos citados dejan entrever que la información que padres y madres de familia tienen respecto al colegio se dinamiza desde la dicotomía: buen colegio y mal colegio. Al profundizar en este aspecto se observa que aparecen como referentes del objeto social colegio dos actores de la comunidad educativa: docentes y estudiantes. Respecto al primero, se retoma lo mencionado por Gadotti (2007) acerca de que "la escuela es el lugar por excelencia para el maestro... estamos acostumbrados a reclamarle a nuestros maestros como si ellos fuesen los responsables de todos los pesares de la humanidad" (p. 9), y asi se ve reflejado en las expresiones de los padres y madres de familia, los docentes son un referente de lo que es el objeto social colegio, toman a este actor para establecer una relación directa con éste y crear una imagen de lo que él representa (colegio), lo cual, puede obedecer a que es el colectivo de docentes el que se encarga de construir la relación con la familia (Martinez, 2014), y se convierte en el eje central porque el colegio es visto desde la función sustantiva de transmisión del saber y bajo la figura de espacio de formalización de la enseñanza (Silveira \& Wagner, 2009; Oliveira, \& Marinho-Araújo, 2010; Pacheco \& Cia, 2015), y esto se ve reflejado desde la misma institucionalidad:

La función docente es aquella de carácter profesional que implica la realización directa de los procesos sistemáticos de enseñanza - aprendizaje, lo cual incluye el diagnóstico, la planificación, la ejecución y la evaluación de los mismos procesos y sus resultados, y de otras actividades educativas dentro del marco del proyecto educativo institucional de los establecimientos educativos. (Decreto 1278, 2002).

Desde la teoría de las representaciones sociales, los elementos presentados hacen parte de la dimensión información, y dejan ver un contenido que refleja lo planteado por Jodelet (2000) en cuanto a la dinámica entre representación y orden social, primero porque en estos elementos es notorio el papel de la interacción social, padres y madres refieren lo que escuchan de sus hijos y de compañeros de ellos desde la cotidianidad vivida en su vinculación con el colegio, segundo, estos elementos están permeados por relaciones entre grupos y relaciones de poder, que para este caso es la institucionalidad, es decir, institucionalmente hay una lógica del rol docente como figura central, y que se ha reproducido desde todo el aparato administrativo que posibilita su acceso y es tomada como referente por padres y madres de familia; $y$ tercero, la historia, puesto que las expresiones dejan manifiesto a los dos actores (docentes y estudiantes) y la función sustantiva sobre 
los cuales se ha erigido el objeto social colegio desde su instauración como sistema de socialización y formación a partir de la división social del trabajo.

Respecto a la dimensión actitud, en el sector rural se encuentra que la imagen favorable del colegio tiene en cuenta elementos como: la disciplina, el inculcar valores, y las actividades complementarias en el proceso de enseñanza aprendizaje, elementos en los que la figura del docente vuelve a aparecer. En el sector urbano aparecen elementos como: la disciplina, las metodologías de enseñanza aprendizaje, la interacción y valoración personal entre profesores y estudiantes y entre grupos de pares, y el tema de la infraestructura física.

Por otro lado, una imagen desfavorable de colegio tiene que ver, en el sector rural, con elementos como: el irrespeto por parte de algunos profesores, la irresponsabilidad de los estudiantes, la irresponsabilidad de las familias, el consumo de sustancias psicoactivas, déficit en el transporte de estudiantes, los chismes y el aseo de las instalaciones. Por su parte en el sector urbano, aparecen elementos como: la indisciplina y el tema académico en los que la figura del docente aparece como referente, la interacción entre pares haciendo alusión a las influencias para el tema de consumo de sustancias psicoactivas y presencia de riñas, vinculando también el tema de infraestructura respecto a temas de seguridad de la zona y deterioro de las instalaciones.

Los resultados corroboran lo manifestado por Martínez (2014) respecto a que familia y escuela mantienen una relación que para algunos puede ser percibida como buena, cordial y fluida, mientras que para otros puede resultar problemática al no darse como se espera; además muestran que en estas instituciones educativas el profesor es un protagonista, cuestión que ha sido referenciada en otros estudios (Cabello \& Giró, 2016), por tanto, el profesor es tomado como referente para la representación de colegio, y además se pone en juego el tema académico, elemento que Silva y Siqueira (2017) identificaron como indicador de conflicto o afinidad entre estudiantes y profesores, y que en este caso particular se traslada a esa relación entre padres y madres de familia y profesores. Estos contrastes permiten interpretar que la función sustantiva (proceso de enseñanza) delegada a la institución colegio y los actores encargados de ella (profesores) se transversalizan en las relaciones de toda la comunidad educativa (profesores, estudiantes y padres de familia), por tanto, se pueden interpretar como elementos constitutivos de una representación social hegemónica (Moscovici, 1988), es decir, son elementos que han trascendido en el tiempo en otros lugares y otros espacios y que los actores a través de la interacción los han hecho propios para crear una imagen de colegio.

De este marco representacional el tema de la participación de padres y madres de familia en el colegio tiene una dinámica particular. Retomando los resultados tenemos expresiones como: "Yo participo cumpliendo y asistiendo a las diferentes actividades y reuniones que se hace en el colegio" (R:15), "yendo a las reuniones asistiendo cuando me llaman al colegio los profesores" (R:2), "Participo biniendo a las reuniones" (U:1), "con el cumplimiento, cuando nos sitan a las reuniones" (U:33). Estas expresiones se relacionan con los elementos representacionales hegemónicos en la medida en que enmarcan el comportamiento de padres y madres de familia en un rol que no va más allá de las reuniones en grupo que se organizan institucionalmente, y puede interpretarse como una relación superficial y limitada a situaciones formales (Reali \& Tancredi, 2002 conforme citados por Oliveira \& Marinho-Araújo, 2010) lo cual puede venir anclado al tema de la trayectoria estudiantil de sus hijos puesto que se ha encontrado en otros estudios que el nivel de participación de las familias es mayor en las etapas formativas inferiores (Gutiérrez \& Alonso, 2011), y para el caso particular del estudio se trata de estudiantes que están en los grados superiores de escuela secundaria, con una mayor trayectoria académica, una etapa de desarrollo particular, y una vinculación institucional más extensa de sus padres y madres.

Sin embargo, este trabajo de investigación permite mencionar que si bien los elementos representacionales tienen consensos entre el contexto rural y urbano también tiene matices, o elementos divergentes, que caracterizan a ambos. Por ejemplo, en el sector rural se encontró expresiones que refieren al tema del espacio físico y la alimentación, y lo cual está muy vinculado a la dinámica actual de la institución referente al plan de alimentación escolar y recursos destinados para la infraestructura: "Un poco me preocupa el restaurante es muy poca la comida" (R:14) "Ampliar sus instalaciones porque hay muchos estudiantes y necesitan un espacio de recreación" (R:13), mientras que en el sector urbano se encontraron expresiones que refieren a una connotación diferenciadora, que aunque mantienen el tema de la formación, le dan un matiz que va un poco más allá de la transmisión de conocimiento, tales como: " el colegio es el recreo de la vida" (U:64), "Que es el colegio es el segundo hogar para nuestros hijos porque forman como personas para el bien de ellos" (U:32), "Este colegio les da la oportunidad para las personas de bajos recursos para que con un buen rendimiento sean alguien en la vida" (U:34).

Al ser las representaciones un conocimiento construido desde la cotidianidad, cada sujeto le insertará elementos que va decodificando en su interacción con el medio y su experiencia propia respecto al mismo. Para el sector rural el tema del espacio físico y la alimentación están generando puntos importantes de quiebre por su dinámica propia respecto a las condiciones que genera 
el contexto, puesto que es conocido que el tema de la extensión educativa y la garantía de condiciones adecuada para ello en los sectores no urbanos es más complejo porque aspectos como la geografía, las condiciones de la escuela y de la misma familia se vinculan de forma determinante (Lima \& Silva, 2015). En el sector urbano estos elementos pueden estar posiblemente cubiertos por una proximidad geográfica hacia la institucionalidad y el factor sociodemográfico, por tanto, hay otros aspectos que median en el discurso de los participantes, pensando un aspecto de tiempo de permanencia de sus hijos en la institución y lo que ha futuro ha de generar todo este proceso en la creación y desarrollo de un mejor proyecto de vida como posibilidad de un buen futuro.

Estos elementos diferenciadores podrían ser señales de la construccion y emergencia de representaciones sociales emancipadas, es decir, aquellas que construyen los subgrupos con versiones propias y diferentes respecto a lo hegemónico, y las cuales pueden favorecer la participación de padres y madres de familia en la relación escuela familia bajo expresiones como " $M$ participación en el colegio es estar pendiente de mis hijos, en su rendimiento... ballan bien presentados con sus uniformes $y$ $q$ lleguen puntualmente" (U: 10), "enseñandoles a mis hijos que lo más importante es el respeto a profesores, compañeros y directivos" (U:53). Esto en función de que una representación diferente de colegio, más allá de su papel institucionalizado, deviene en un marco diferente de comportamiento.

\section{CONSIDERACIONES FINALES}

Los resultados presentados permiten aproximarnos al engranaje psicosocial que subyace en la relación escuela y familia, "nos da ciertas interpretaciones, que son pequeñas teorías" (Jodelet 2002, conforme citada por Esparza, 2003, p. 125) sobre la imagen que se crea acerca del objeto social colegio y que desde los diferentes contextos, realidades y sentires se vienen gestando y que les permite a padres y madres de familia tomar posición respecto a dicho objeto social.

"Si partimos de que una representación social es una preparación para la acción, no lo es solo en la medida en que guía el comportamiento, sino sobre todo en la medida en que remodela y reconstituye los elementos del medio en el que el comportamiento debe tener lugar" (Moscovici, 1979, p. 32) al tomar los resultados como elementos representacionales es posible inferir un marco de comportamientos y comunicación en los sujetos, de ahí, que se hayan inferido dos tendencias representacionales: hegemónicas y emancipadas, las cuales pueden dar cuenta de la naturaleza de estímulos que están rodeando y provocando la generación de conocimiento de sentido común de los sujetos, y que a su vez les permite el intercambio de códigos para denominar y clasificar su historia individual y colectiva respecto al objeto social.
Una imagen favorable de colegio puede devenir en un marco de comportamientos potenciadores de la relación escuela familia, sin embargo, el mismo va a depender de la hegemonía o emancipación construida respecto al objeto social, es decir, el sujeto desde esa imagen de favorabilidad puede reproducir esa lógica institucionalizada donde el colegio se fundamenta en su función sustantiva de transmisión de conocimiento con preponderancia del rol docente, o por el contrario puede, desde una misma imagen favorable, promover nuevas formas de interacción con el objeto social, tal vez, tomando un rol menos subordinado a la figura del docente; y de forma similar desde una imagen de des favorabilidad. Esto a la luz del contexto, la historia y las relaciones de poder que se han venido gestando entre la escuela y la familia, elementos cristalizados a través de las interacciones en la cotidianidad.

Los elementos encontrados permiten ver que si bien pueden existir consensos también existen divergencias respecto al objeto social, por tanto, esto da lugar a cuestionar aquellas lógicas institucionalizadas (tanto en la escuela y la familia) en las que los roles establecidos son rígidos e invisibilizan matices representacionales, lo cual, deviene en una homogenización de prácticas, que si bien pueden regular ciertos aspectos, pueden también desdibujar la relación entre escuela y familia. En este orden de ideas, fortalecer todo el tema de la corresponsabilidad se considera relevante en un proceso de intervención.

Finalmente, al ser un estudio exploratorio, los resultados pretenden ser un disparador para profundizar en el tema del conocimiento común que padres y madres de familia construyen sobre el objeto social colegio, puesto que para dar cuenta del campo de representación (elemento que hace parte del contenido representacional) se requiere ahondar en ellos y hacer uso de múltiples técnicas, cuestión en la cual el presente estudio se limita.

\section{REFERENCIAS}

Barboza-Palomino, M.; Moori, I.; Zárate, S.; López, A.; Muñoz, K.; Ramos, S. (2017). Influencia de la dinámica familiar percibida en el proyecto de vida en escolares de una institución educativa de Lima. Psicologia Escolar e Educacional, 21(2), 157-166.

Bustamante, L. (2010). Representaciones de los padres acerca de la escuela primaria. Creencias, opiniones, valoraciones, e imágenes sobre la "buena escuela" y la "escuela ideal". Congreso Iberoamericano de educación Metas 2021. Buenos Aires, República Argentina.

Cabello, S.; Giró, J. (2016). El papel y la representación del profesorado en la participación de las familias en la escuela. Revista electrónica interuniversitaria de formación del profesorado, 19(1), 61-72.

Cabrera, V.; Aya, V.; Muñoz, D.; Guevara, I.; Cano, A. (2016). Madres, padres y profesores como educadores de la 
resiliencia en niños colombianos. Psicologia Escolar $e$ Educacional, 20(3), 569-579.

Callegaro, J.; Wanderley, G.; Koller, S. (2015). A participação dos pais nas pesquisas sobre o bullying escolar. Psicologia Escolar e Educacional, 19(1), 41-48.

Castro-Zubizarreta, A.; Garcia-Ruiz, R. (2016). Vínculos entre familia y escuela: visión de los maestros en formación. Magis, revista internacional de investigación en educación, 9(18), 193-208.

Decreto 1278, de 19 de junio de 2002 (2002, 19 de junio). Por el cual se expide el Estatuto de Profesionalización Docente. Colombia: Ministerio de Educación de Colombia.

De Freitas, T. (2008). Entre escolas e famílias, revelações dos deveres de casa. Paidéia, 18(40), 385-398.

Silveira, L. M. O. B.; Wagner, A. (2009). Relação família-escola: práticas educativas utilizadas por pais e professores. Psicologia Escolar e Educacional, 13(2), 283-291.

Diaz, A.; Salamanca, L. (2012). Los jóvenes son sujetos políticos... a su manera. Utopía y praxis latinoamericana, 17(57), 109-117.

Esparza, L. (2003). Entrevista a Denis Jodelet, realizada el 24 de Octubre de 2002 por Oscar Rodriguez Cerda. Relaciones. Estudios de Historia y sociedad, 24(93), 117-132.

Gadotti, M. (2003). Historia de las ideas pedagógicas. México: Siglo XXI.

Gadotti, M. (2007). La escuela y el maestro, Paulo Freire y la pasión de enseñar. Caracas, Venezuela: Centro Internacional Miranda.

Gutiérrez, C.; Alonso, J. (2011). Relevancia de la formación y participación en la relación familia-escuela en el marco de la evaluación de los proyectos para la participación de la familia en Canarias. International journal of developmental and educational psychology, 3(1), 545-554.

Jodelet, D. (1993). La representación social: fenómenos, concepto y teoría. In Moscovici (Ed.), Psicología social II, pensamiento y vida social, pensamiento social y problemas sociales (pp. 469-494). Barcelona: Paidós.

Jodelet, D. (2000). Representaciones sociales: contribución a un saber sociocultural sin fronteras. In Jodelet, D.; Guerrero, A. (Eds.), Develando la cultura, estudios en representaciones sociales (pp. 7-30). México: Universidad Nacional Autónoma de México.

Jodelet, D. (2011). Aportes del enfoque de las representaciones sociales al campo de la educación. Espacios en blanco
Revista de educación, 21(1), 133-154.

Julio, V.; Manuel, M.; Navarro, L. (2012). Estrategia educativa para la participación de los padres en compromisos escolares. Escenarios, 10(2), 119-127.

Martínez, S. (2014). Familias y escuelas en tres centros educativos: en busca de relaciones compartidas. Profesorado, revista de curriculum y formación de profesorado, 18(2), 117-133.

Moscovici, S. (1979). El psicoanálisis, su imagen y su público (N. Finetti, Trad.). Buenos Aires: Ed Huemul.

Moscovici, S. (1988). Notes towards a description of Social Representations. European Journal of Social Psychology, $18,211-250$

Moscovici, S. (2000). Social representations. Explorations in social psychology. (Duveen, G., Ed.) Cambridge: Polity.

Oliveira, C. \& Marinho-Araújo, C. (2010). A relação famíliaescola: intersecções e desafios. Estudos de Psicologia, 27(1), 99-108.

Pacheco, A.; Cia, F. (2015). Educação especial e a relação família-escola: análise da produção científica de teses e dissertações. Psicologia Escolar e Educacional, 19(2), 349-358.

Lima, L. P.; Silva, A. P. S. (2015). A relação entre a educação infantil e as famílias do campo. Psicologia Escolar $e$ Educacional, 19(3), 475-483.

Silva, V.; Siqueira, M. (2017). La relación profesor-alumno en la enseñanza secundaria: percepción del profesor de escuela pública. Psicologia Escolar e Educacional, 21(2), 245-252.

Suarez, J.; Urrego. L. (2014). Relación familia-escuela: una mirada desde las prácticas pedagógicas rurales en Anserma Caldas. Revista Latinoamericana de estudios de familia, 6, 97-113.

Tyrell, H.; Vanderstraeten, R. (2017). Familia y escuela: algunas reflexiones sobre la diferenciación interna de la educación. Revista Mad, revista del magister en análisis sistémico aplicado a la sociedad, (36), 1-20.

Araújo, P. V.; Pessoa, V. S., V.; Fonseca, P. N.; Albuquerque, J. H. A.; Almeida, A. C. (2016). Eu gosto da escola: um estudo sobre o apego ao ambiente escolar. Psicologia Escolar $e$ Educacional, 20(2), 377-384.

Villarroel, G.; Sánchez, X. (2002). Relación familia y escuela: un estudio comparativo en la ruralidad. Estudios pedagógicos, (28), 123-141.

Agradecimiento a la Corporación Universitaria Minuto de Dios (Colombia) por la financiación, a través de la VI Convocatoria para el desarrollo y fortalecimiento de la investigación en UNIMINUTO, del proyecto de investigación titulado: el colegio desde las representaciones sociales construidas por padres y madres de familia, del cual se deriva el contenido del presente artículo. 